\title{
An Experimental Study of Drag Reduction in a Pipe with Superhydrophobic Coating at Moderate Reynolds Numbers
}

\author{
J.-H. Chen ${ }^{1, a}$, C.-C. Tsai ${ }^{1}$, Y.-Z. Kehr ${ }^{1}$, L. Horng ${ }^{2}$, K. Chang ${ }^{2}$, and L. Kuo ${ }^{2}$ \\ ${ }^{1}$ National Taiwan Ocean University, Department of Systems Engineering and Naval Architecture, 2 \\ Pei-Ning Road, Keelung, Taiwan. \\ ${ }^{2}$ Gelwell Biotech Corp., 27-5 Jungjeng E. Rd., Sec. 2, Danshuei Jen, Taipei County, Taiwan.
}

\begin{abstract}
This paper experimentally investigates drag reduction, durability for operations and effects for preventing microorganism from adhering to the surface when the superhydrophobic coating is applied on a solid surface. The experiments are divided into two parts. In the first part, a pipe flow system was established to measure the drag and to test the durability of the micro-structure of superhydrophobic coating at average speeds varying from $1 \mathrm{~m} / \mathrm{sec}$ to $6 \mathrm{~m} / \mathrm{sec}$. In the second part, we tested the effect for preventing microorganism from adhering to the surface by putting the coated steel plates into sea water. There are four different superhydrophobic coatings in the present study. The experimental results were compared to those applied by ship paint usually used at CSBC.
\end{abstract}

\section{Introduction}

In engineering applications, drag reduction by a superhydrophobic coating has been an important issue in recent years. The physical significance behind the popular issue is the fact that a limited slippage of fluid particles adjacent to a solid surface with a coating of superhydrophobic material becomes possible.

In the past few centuries, physicists in fluid mechanics have justified that on a solid boundary, the viscous fluid would have zero velocity relative to the boundary from the macroscopic point of view. This is the no-slip condition which is well known to people in fluid mechanics. Though rigorous studies show that slippage of fluid particles on a solid surface is possible as revealed in molecular dynamics, such a slippage is usually restricted within the nano-scale [1]. The implication of the no-slip condition is the generation of viscous dissipation which occurs as fluid particles flow past the surface and brings them to rest due to roughness or irregularities of the surface. The viscous dissipation manifests macroscopically itself in the form of frictional resistance which makes a significant contribution to total resistance.

Nevertheless, recent studies reveal that reducing the effective contact area of fluid particles on a solid surface leads to a significant reduction of the frictional resistance [2,3]. This is particularly achievable through a textured superhydorphobic surface with a regular or irregular pattern because above it a thin air layer is entrapped and liquid particles move partly on the air layer to form a liquid-

\footnotetext{
a e-mail : jhchen202@gmail.com
} 
air interface. Therefore, the traditional no-slip boundary condition is no longer applicable and a limited slippage of fluid particles is observed [3]. Byun et al. [4] fabricated microgrooves along vertical walls to form superhydrophobic surfaces and measured velocity profiles by microparticle image velocimetry. A slip length of approximately $2 \mu \mathrm{m}$ was observed. Ou and Rothstein [5] demonstrated experimentally that slip along the shear-free liquid-air interface was the primary mechanism responsible for the drag reduction observed for flows over superhydrophobic surfaces.

There are two ways to fabricate a superhydrophobic surface. The first one is to create a rough surface, for example, through the nanolithography technique. It produces a well-organized pattern. Henoch et al. [6] employed the deep reactive ion etching to form the nanograss structure. Their experimental data show that a drag reduction up to $50 \%$ was observed for laminar flow, compared to that by traditional laminar-boundary-layer flow prediction. However, at the onset of turbulent flow, the drag rises sharply and soon becomes substantially higher. Truesdell et al. [7] conducted laminar Couette flow measurements near a regularly textured superhydrophobic surface and drag reduction on the order of $20 \%$ was achieved. Recently, Daniello et al. [8] developed superhydrophobic surfaces with regular arrays of microridges aligned in the flow direction. They employed the particle image velocimetry and pressure drop measurements to demonstrate that periodic, micropatterned superhydrophobic surfaces could reduce drag in the turbulent regime. Nevertheless, the nanolithography technique is usually restricted to the laboratory scale. No practical industry-scale applications, such as in marine transportation, have been reported in the literature.

The second way to form a superhydrophobic surface is to modify the surface, for example, with coating. In most early studies, polymer coatings were employed for drag reduction. Jones and Thurston [9] applied non-newtonian soluble polymer coating on surfaces of underwater vehicles. The frictional resistance was reduced 30 percent in freshwater and 27 percent in seawater. McCormick et al. [10] employed high molecular weight, water-soluble copolymers and conducted a series of drag reduction tests. Their results showed that the effectiveness of drag reduction was dependent on polymer structures and polymer-solvent interactions. In addition, Watanabe et al. [11, 12] carried out experiments on highly water-repellant walls formed by the coating of a fluorinealkane-modified acrylic resin with added hydrophobic silica. The coating resulted in a hydrophobic surface crisscrossed by microcracks of $10-20 \mu \mathrm{m}$ in width. Pressure drop and velocity profile measurements demonstrated drag reduction up to $18 \%$ and slip lengths up to $450 \mu \mathrm{m}$ for flows at Reynolds numbers between 500 and 10,000.

The superhydrophobic nano-coating technology was developed in the past decade. In fact, nanostructures on a solid surface are essential for superhydrophobicity because they induce a high contact angle. Various experimental attempts have been made to create a superhydrophobic surface for drag reduction [13-17]. However, most methods are complicated in the sense that they usually need several steps, special instruments, high temperature and/or low surface energy material modification, which make it difficult for practical applications in large-area superhydrophobic surfaces.

In the present study, we set up a pipe flow system. The inner side of the pipe was coated with the superhydrophobic paint developed by Gelwell Biotech Corp. In contrast to most coatings reported in the literature, the coating in the present study can be applied at the room temperature without special instruments and needs only one step to complete. Tests of durability and drag reduction were carried out. In addition, several plates were coated with the paint and immersed in seawater to test the capability of anti-fouling which is an important issue for seagoing vehicles.

\section{Experimental Setup}

The reduction of drag results from the air layer entrapped in the nanostructure of the coated surface. For practical engineering applications, it is vital to keep the nanostructure intact and air effectively retained under long-term washing of fluid flow. In the present experimental study, we employed the superhydrophobic coating formula developed by Gelwell Biotech Corp. on the test surfaces. 
The coating was applied on surfaces of steel pipes and steel plates. The pipes were employed for the test of drag reduction and durability. The plates were used for the test of anti-fouling capability. Three layers of anti-corrosive primers were first coated on the surfaces before the superhydrophobic coating was applied. For comparisons, we also conducted tests of the pipes and plates with the standard coating of CSBC Corp., Taiwan which includes three layers of anti-corrosive primers and four layers of self polishing anti-fouling paints.

There are five superhydrophobic coating formulae in our study. Four of them were applied on pipes for the drag reduction tests. They were Type A, B, C, and D. Table 1 shows the relative characteristics of the coating surfaces. And four of them were applied on the steel plates. They were Type B, C, D, and E. Table 2 briefly describes the relative characteristics of coating surfaces.

The experiments for each test are briefly described in the following.

\subsection{Tests of drag reduction and durability}

Figure 1 shows the setup of recirculating pipe flow system for tests of drag reduction and durability. The two water tanks were filled with water. A steel pipe of $3.6 \mathrm{~m}$ long was connected on one end to one water tank and on the other end to a flow meter which was then connected to a motor. The inner diameter of the pipe was $34.2 \mathrm{~mm}$. The temperature of water was $24-26^{\circ} \mathrm{C}$ at the onset of tests and we kept it constant during the experiment. A plastic pipe was connected to the outlet of the motor and the water was then shed into the second tank. The outlet of the plastic pipe was immersed in the water of the second tank. Another plastic pipe of much larger diameter connected two tanks at their bottoms so that the water could flow back to the first tank.
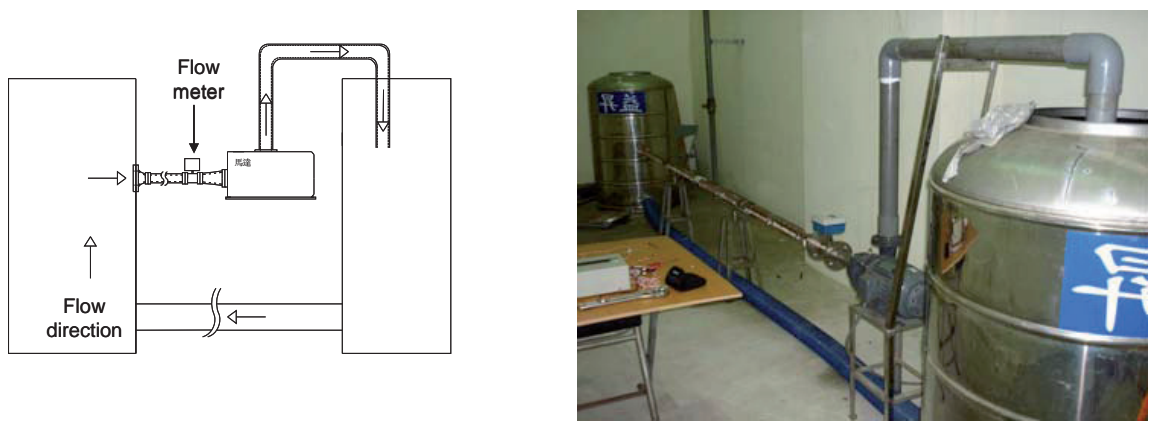

Fig. 1. Setup for pipe flow tests.

A transformer was employed to adjust the motor speed. Figure 2 shows the calibration curve of the motor speed to the average flow speed for the pipe with CSBC standard coating. It is obvious that the relation between them is linear for the motor speed less then $36 \mathrm{rps}$. Due to the loading problem of the motor, no more water can be pumped at a motor speed higher than $36 \mathrm{rps}$.

There were three observing acrylic windows on the pipe in order to monitor the flow. Tests and observations were carried out at six different average flow speeds, ranging from $1 \mathrm{~m} / \mathrm{sec}$ to 6 $\mathrm{m} / \mathrm{sec}$. All the flows were turbulent.

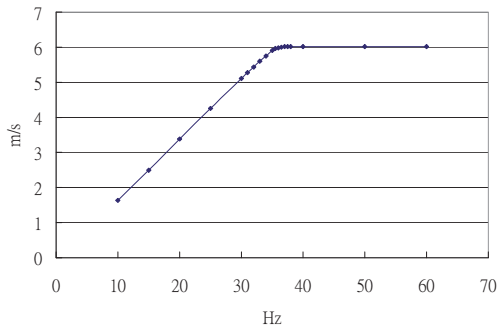

Fig. 2. Calibration of motor and flow speeds. 
EPJ Web of Conferences

Table 1. Characteristics of nanostructures for different coating formulae for drag reduction tests.

\begin{tabular}{c|ccc}
\hline & Clearance volume & Scale & Uniformity \\
\hline Type A & Small & Small & Uniform \\
Type B & Smaller & Small & More uniform \\
Type C & Bigger & Big & Least uniform \\
Type D & Big & Big & Less uniform \\
\hline
\end{tabular}

Table 2. Characteristics of nanostructures for different coating formulae for anti-fouling tests.

\begin{tabular}{c|ccc}
\hline & Clearance volume & Scale & Uniformity \\
\hline Type B & Smaller & Small & More uniform \\
Type C & Bigger & Big & Least uniform \\
Type D & Big & Big & Less uniform \\
Type E & Small & Small & Uniform \\
\hline
\end{tabular}

\subsection{Tests of Anti-fouling}

For the test of anti-fouling, two steel plates of $30 \mathrm{~cm} \times 30 \mathrm{~cm}$ were prepared. On one side of each plate was coated with the standard paints of CSBC Corp., Taiwan and on the other side the superhydrophobic paints of four different formulae (Type B, C, D, and E) developed by Gelwell Biotech Corp. Figure 3 shows the two sides of the plate.

The two plates were then immersed in the seawater at a pier of CSBC Keelung Shipyard, as shown in Figure 4. To avoid collisions due to wave motions, the depths of the two plates in the water are different. One of the plates, labelled as Plate A, was pulled out of the water every day and

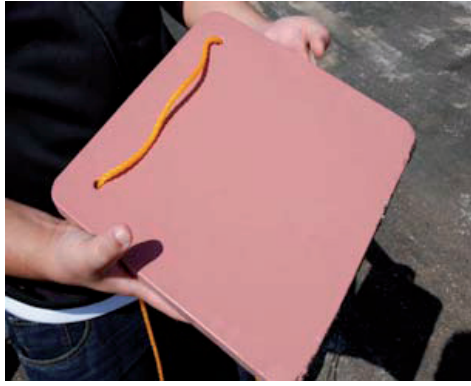

(a) The side for CSBC paint.

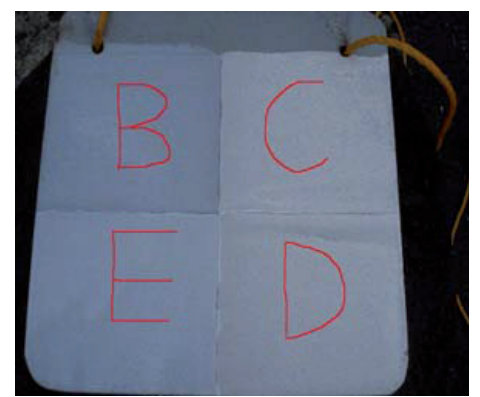

(b) The side for superhydrophobic paints.

Fig. 3. Setup for anti-fouling tests.

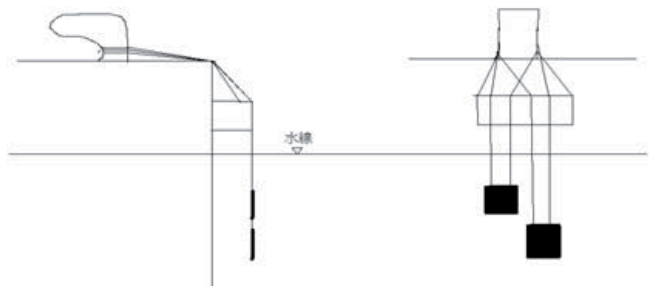

(a) Schematic for anti-fouling test.

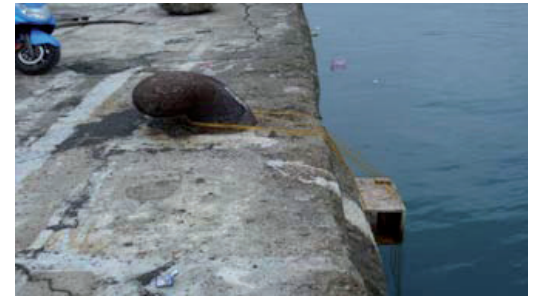

(b) The site for anti-fouling tests.

Fig. 4. Site arrangement for anti-fouling test. 


\section{4th International Conference on Experimental Mechanics}

exposed to the air for 20-30 minutes; the other plate, labelled as Plate B, was pulled out of the water every two days and exposed to the air for 20-30 minutes. After exposition to the air, they were immersed again in the seawater. The whole test lasted for a month.

\section{Some Results and Discussions}

\subsection{Tests of drag reduction and durability}

Four types of coating formulae were applied on four different pipes. They were labelled as Type A, $\mathrm{B}, \mathrm{C}$, and D. Their nanostructures were qualitatively described in Table 1 . The test speeds vary from $1 \mathrm{~m} / \mathrm{sec}$ to $6 \mathrm{~m} / \mathrm{sec}$. These test speeds are much higher than those available in literature and more practical as far as industrial applications in the real world are concerned. The corresponding Reynolds numbers vary from about $3.8 \times 10^{4}$ to $2.3 \times 10^{5}$. Obviously, the flows in all of our tests lie in the regime of turbulence.

The test for each coating formula started at $1 \mathrm{~m} / \mathrm{sec}$. Two standard runs of tests were conducted. For the first short run of test, the average flow speed was increased by $1 \mathrm{~m} / \mathrm{sec}$ for every $30 \mathrm{sec}$ till it reached $6 \mathrm{~m} / \mathrm{sec}$. Then for the second run of test, we conducted the long-term test. Again, we began the test at the flow speed of $1 \mathrm{~m} / \mathrm{sec}$. However, the speed was increased by $1 \mathrm{~m} / \mathrm{sec}$ every 60 minutes. The variation of motor speed was monitored during the test and taken every second by a personal computer. The motor speed is an indicator of power required to drive the flow at a specific speed. Obviously, a higher motor speed for a specific flow speed implies higher drag at that flow speed.

Several interesting phenomena have been observed in these tests.

At the onset of the flow test with coating formula A, we found that for an average flow speed higher than $5 \mathrm{~m} / \mathrm{sec}$, the power required almost leveled off, as shown in Figure 5(a). It implies that the flow can be easily accelerated with almost no additional power owing to some kind of physical interaction between the fluid and nanostructure which is not clear yet. This is in sharp constrast to the situation for a flow speed less than $5 \mathrm{~m} / \mathrm{sec}$. For the latter, the motor speed grew almost linearly with the flow speed.

In addition, because the required power levels off at a higher speed, the growth of drag drastically reduced as the speed was increased, compared to that due to CSBC standard coating. Consequently, the drag on the surface with the superhydrophobic coating was even less than that due to CSBC standard coating at a flow speed higher than $5.8 \mathrm{~m} / \mathrm{sec}$. It is quite interesting that the superhydrophobicity of the coating shows its due characteristic of drag reduction at a higher speed for which turbulent effects are supposed to be more significant.

Nevertheless, such a good feature did not keep too long in time before the nanostructure was somehow destroyed and the required power was substantially increased as the speed of flow was

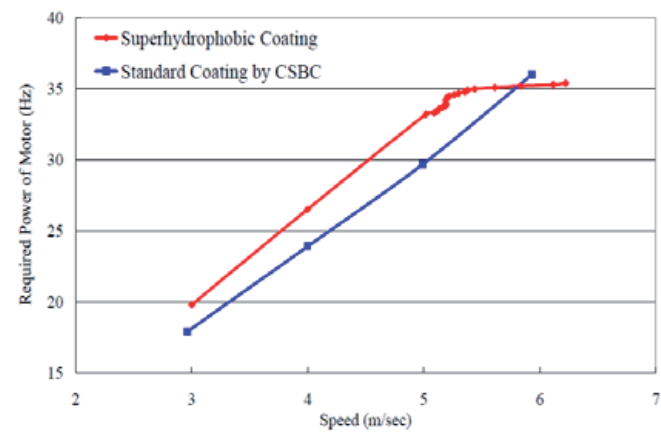

(a) First 30 minutes of test.

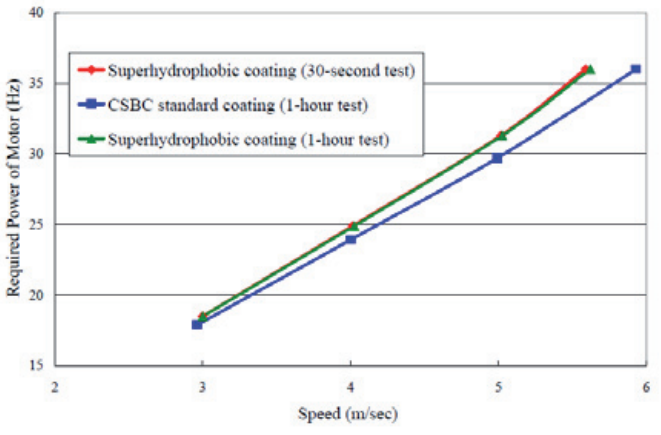

(b) After 30 minutes of test.

Fig. 5. Power required for superhydrophobic coating formula A and CSBC standard coating formula. 
raised at a high speed. In fact, the significant feature of drag reduction at a higher speed shown in Figure 5(a) disappeared after about 30 minutes. Figure 5(b) shows the abrupt change of the powerspeed curve. Nevertheless, the difference between the two curves becomes smaller.

The superhydrophobicity of the coated surface before and after the tests of about 24 hours is shown in Figure 6. Obviously, the surface has been somehow and somewhat destroyed and the superhydrophobicity was not so perfect as that before the test. This might explain why the drag during the test had an abrupt change. Nevertheless, the mechanism of such an abrupt change is not clear and further study of nanostructure is required.

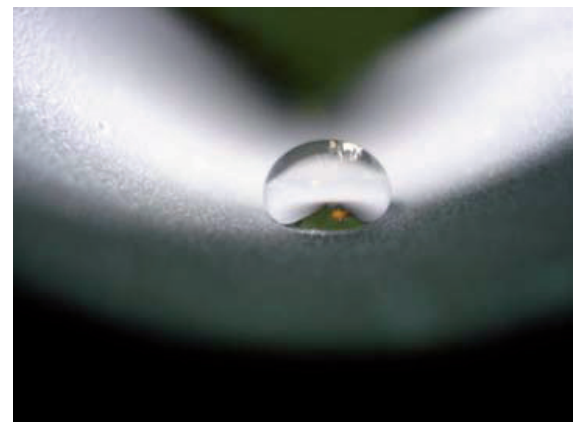

(a) Before the test.

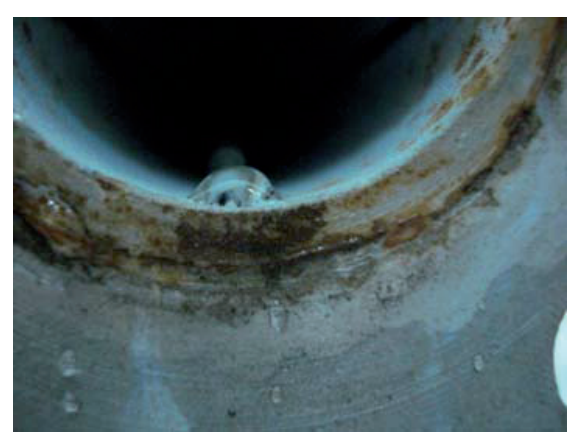

(b) After the test.

Fig. 6 Superhydrophobicity of the surface coated by formula A.

For other formulae, the results are shown in Figure 7. General observation shows that the drag was not reduced for the surface with any of the four superhydrophobic coating for-mulae at a speed higher than $1 \mathrm{~m} / \mathrm{sec}$. Nevertheless, at the speed of $1 \mathrm{~m} / \mathrm{sec}$, the resistances of different coatings are almost identical. It appears that the drag of the present superhydrophobic coatings is smaller than that of the CSBC standard coating. This observation is similar to the results concluded by Henoch et al. [6].

Furthermore, the uniformity of the nanostructure due to coating does affect the drag. This can be

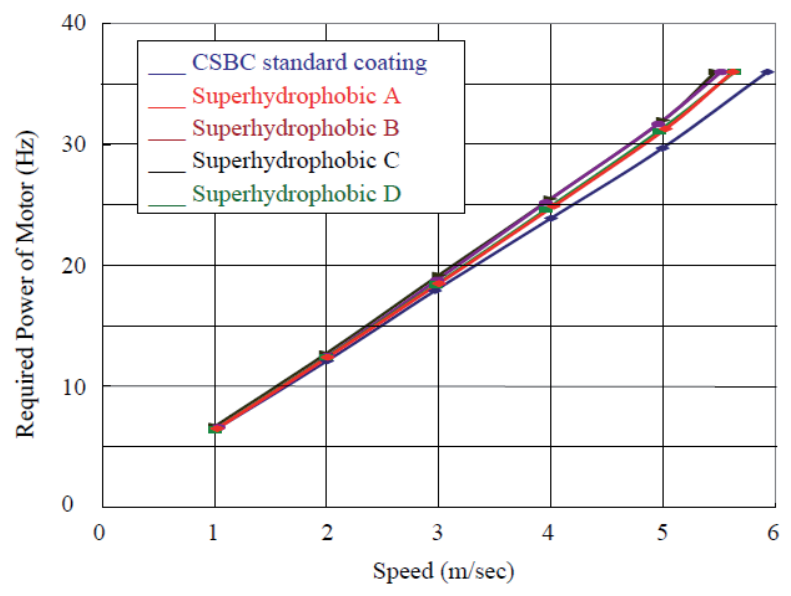

Fig. 7 Power required for various coating formulae. observed in Figure 7. Therefore, the development of the coating technology to create a uniform surface structure is important in engineering applications of drag reduction.

\subsection{Tests of Anti-fouling}

Before immersing the plates in the seawater, we conducted a superhydrophobic test. Shown in Figure, the coated surface exhibits its perfect superhydrophobicity.

Four test days later, the surfaces with formulae C and D still show partial superhydrophobic property for Plate A. However, the property of superhydrophobicity does not sustain for other parts of Plate A and all parts of B. This may imply that the seawater diffused into the air layer entrapped 
in the textured superhydorphobic surface and it became wet. These are shown in Figures 9 and 10 . The test shows that the surface regularly exposed to air is important to keep it superhydrophobic.

Otherwise, the property of superhydrophobicity can soon be destroyed not too long after the surface becomes wet. Nevertheless, the required time interval of air exposition may vary for surface coated with different coating formulae.

The experiment shows that the surfaces of Type $\mathrm{C}$ and $\mathrm{D}$ can sustain longer before the seawater diffuses into the air layer. It is interesting to find that these two types of coating resulted in a nanostructure of less uniformity and larger scale.

For Plate B, the fouling appeared on the superhydrophobic surfaces 4 days later. Furthermore, the fouling can be observed on the side with CSBC standard

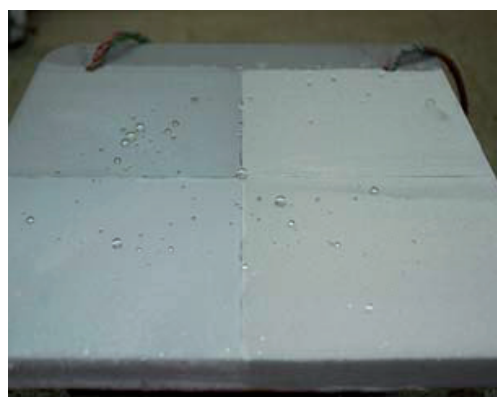

(a) Top view.

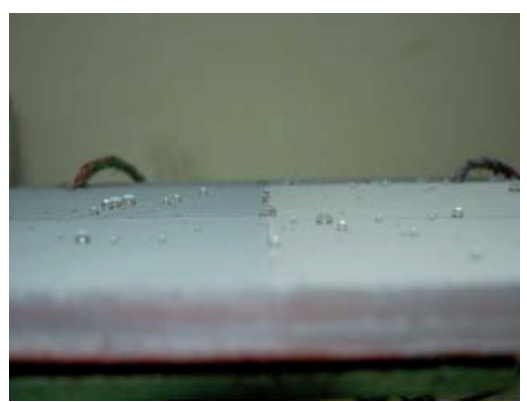

(b) Side view.

Fig. 8 Superhydrophobicity test of the surface coated by different formulae.

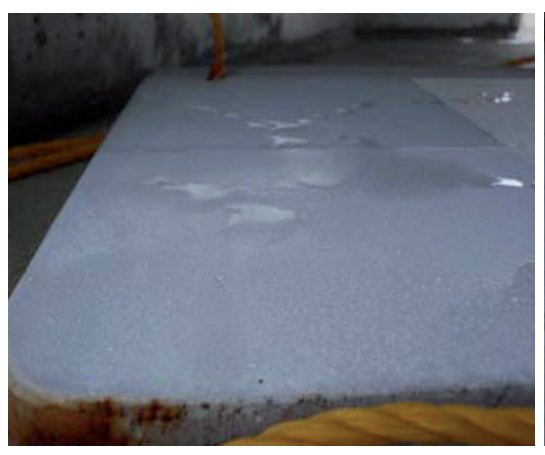

(a) Type B and E.

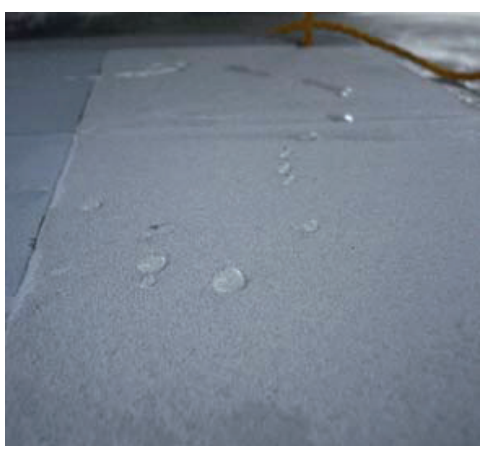

(b) Type C and D.

Fig. 9 Superhydrophobicity test of Plate A after 4 days of seawater immersion.

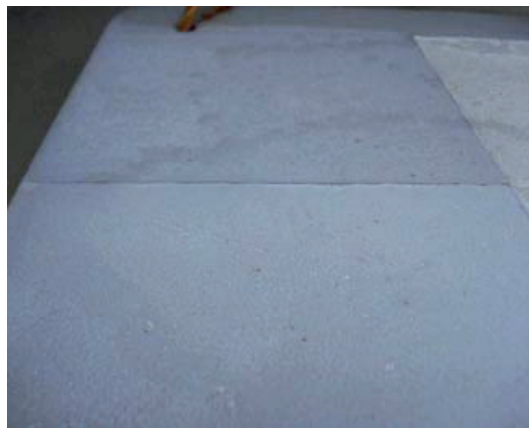

(a) Type B and E.

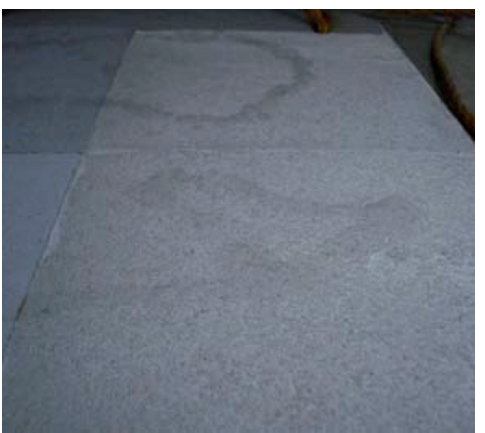

(b) Type C and D.

Fig. 10 Superhydrophobicity test of Plate B after 4 days of seawater immersion. coating after 8 days of immersion test.

For Plate A, we could expect that the anti-fouling capabi-lity could be better. In fact, the fouling appeared on the superhydrophobic surfaces 11 days later. Furthermore, the fouling can be observed on the side with CSBC standard coating after 18 days of immersion test.

It appeared that longer superhydrophobic sustainability results in better anti-fouling capability. The loss of superhydrophobicity results in the fouling phenomenon on surfaces with superhydrophobic coating much earlier than that with CSBC standard coating. 


\section{Conclusions}

A series of tests of drag reduction and anti-fouling capability of surfaces with superhydrophobic coating were conducted.

The drag reduction tests were carried out at speed varying from $1 \mathrm{~m} / \mathrm{sec}$ to $6 \mathrm{~m} / \mathrm{sec}$. The test speeds are much higher than those available in the literature. Some peculiar phenomena were observed. For some surface with the superhydrophobic coating, the drag reduction could be achieved at the early stage of the test. However, the speed-power curve abruptly changed and the effect of drag reduction disappeared. The mechanism which induced such an abrupt change is not clear. A further study is needed. Furthermore, the uniformity of the surface nanostructure does affect the drag. A uniform structure results in a smaller drag.

The anti-fouling tests show that the superhydrophobic surface need be regularly exposed to air to keep its superhydropobicity and, hence, anti-fouling capability. Furthermore, it appears that longer superhydrophobic sustainability results in better anti-fouling capability. This conclusion contradicts with that of the drag reduction. However, this could be overcome if the time interval of the regular air exposition is reduced.

\section{Acknowledgements}

The present study was made possible by the financial support of Gelwell Biotech Corp. and National Science Council of the Republic of China under the grant NSC 97-2622-E-019-004-CC3 and the provision of CSBC standard paint and the anti-fouling test site from CSBC. The authors would like to express their thanks to these supports.

\section{References}

1. C.-H.Choi, K.J.A. Westin, K.S. Breuer, Phys. Fluids, 15, 2897 (2003).

2. J. Ou, B. Perot, J.P. Rothstein, Phys. Fluids, 16, 4635 (2004).

3. S. Gogte, P. Vorobieff, R. Truesdell, A. Mammoli, F. Van Swol, P. Shah, C.J. Brinker, Phys. Fluids, 17, 051701 (2005).

4. D. Byun, J. Kim, H.S. Ko, H.C. Park, Phys. Fluids, 20, 113601 (2008).

5. J. Ou, J.P. Rothstein, Phys. Fluids, 17, 103606 (2005).

6. C. Henoch, T.N. Krupenkin, P. Kolodner, J.A. Taylor, M.S. Hodes, A.M. Lyons, C. Peguero, K. Breuer, 3rd AIAA Flow Control Conf. (2006).

7. R. Truesdell, A. Mammoli, P. Vorobieff, F. van Swol, C. J. Brinker, Phys. Rev. Lett. 97, 044504 (2006).

8. R.J. Daniello, N.E. Waterhouse, J.P. Rothstein, Phys. Fluids, 21, 085103 (2009).

9. R.D. Jones, S. Thurston, J. Aircraft, 2, 122 (1965).

10. C.L. McCormick, R.D. Hester, S.E. Morgan, A.M. Safieddine, Macromolecules, 23, 2124 (1990).

11. K. Watanabe, T. Takayama, S. Ogata, S. Isozaki, AIChE J., 49, 1956 (2003).

12. K. Watanabe, Y. Udagawa, H. Udagawa, J. Fluid Mech., 381, 225 (1999).

13. X. Lu, C. Zhang, Y. Han, Macromol. Rapid Commun., 25, 1606 (2004).

14. L. Jiang, Y. Zhao, J. Zhai, Angew. Chem. Int. Ed., 43, 4338 (2004).

15. L. Zhai, F.C. Cebeci, R.E. Cohen, M.F. Rubner, Nano Lett., 4, 1349 (2004).

16. H.Y. Erbil, A.L. Demirel, Y. Avci, O. Mert, Science, 299, 1377 (2003).

17. Q. Xie, G. Fan, N. Zhao, X. Guo, J. Xu, J. Dong, L. Zhang, Y. Zhang, C.C. Han, Adv. Mater., 16, 830 (2004). 